\title{
A New Target Polarization Measurement System for the Fermilab Polarized Drell-Yan SpinQuest Experiment
}

Mikhail Yurov*

Los Alamos National Laboratory

E-mail: myurovdLanL.gov

Patrick L. McGaughey

Los Alamos National Laboratory

E-mail: pLmdLan1.9ov

Jacqueline Mirabal-Martinez

Los Alamos National Laboratory

E-mail: Gmirabaldlan1.9ov

The Liverpool Q-meters were developed in the late 70s and became a de facto industry standard for NMR-based polarization measurements of polarized solid targets. However, it is becoming increasingly more difficult to produce the required number of q-meter channels as the components have become obsolete. The Los Alamos National Laboratory (LANL) group has developed a new NMR-based polarization measuring system following the basic Liverpool design. The new Q-meter will have multiple improvements, such as remote tuning and compact design. These improvements present opportunities for achieving a higher figure of merit for experiments exploiting polarized solid targets by potentially increasing the accuracy of the polarization measurements. The new LANL Q-meter is intended to be used in Fermilab SpinQuest/E1039 experiment which is part of the continuing world-wide effort to shed light on the nucleon spin composition puzzle. The current status of this work is presented.

The 18th International Workshop on Polarized Sources, Targets, and Polarimetry, PSTP2019 23-27 September, 2019

Knoxville, Tennessee

${ }^{*}$ Speaker. 


\section{Introduction}

As for many scattering experiments exploring spin structure of the nucleon, the usage of the solid dynamically polarized (DNP) target became an optimum solution for the Fermilab SpinQuest (E1039) experimental program. The SpinQuest experiment [U] proposed by LANL is expected to perform the first high statistics measurement of the sea quarks Sivers function. The Sivers function is formulated within the framework of transverse momentum dependent distribution (TMD) functions which explore the correlation of quark motion relative to the nucleon spin direction. Among the eight leading TMDs, the Sivers function represents the transverse momentum distribution of unpolarized quarks in a transversely polarized nucleon. The ultimate goal of the experiment is to provide an unambiguous indication of whether the light anti-quarks angular orbital momentum makes a contribution to the nucleon spin. Experimentally, the sea quarks Sivers asymmetry can be accessed in proton-proton scattering through polarized Drell-Yan (DY) production. In comparison to other mechanisms sensitive to Sivers function such as Semi Inclusive Deep Inelastic Scattering (SIDIS), the DY process offers great kinematical selectivity for sea quarks and allows a direct Sivers function measurement without the complications of fragmentation functions and final state interactions.

The measurements will be carried out using the Fermilab main injector unpolarized proton beam with an energy of $120 \mathrm{GeV}$ interacting with transversely polarized proton and neutron targets. The azimuthal distribution of dimuons produced in DY events will be analyzed by the existing SeaQuest (E906) Muon Spectrometer. It is proposed to determine the size and the sign of the Sivers function for both $\bar{u}$ and $\bar{d}$ quarks. The experiment will explore a unique range of virtualities $\left(Q^{2} \sim 10 \mathrm{GeV}^{2}\right)$ and transverse momenta $\left(q_{T}\right.$ in the few $\mathrm{GeV}$ region) currently inaccessible through other measurements and measure the Sivers asymmetry for four different Bjorken, $x_{B}$, bins in the range $0.1<x_{B}<0.5$. The ability to conduct the experiment at maximum luminosity and nucleon polarization is paramount to achieving these goals. Therefore, a specific type of solid polarized target system, which during this workshop was proposed to be called the D. Crabb target [Q, (1], 团], was constructed by the LANL and UVA polarized target groups for the E1039 experiment.

The distinct features of the $D$. Crabb target present in the E1039 polarized target system include, first, the usage of ammonia $\left(\mathrm{NH}_{3}\right)$ and deuterated ammonia $\left(N D_{3}\right)$ as a choice of the target material. Secondly, the vertically pointing $5 \mathrm{~T}$ magnetic field is provided by a split pair superconducting magnet and the target material is cooled to $\sim 1 \mathrm{~K}$ with a high cooling capacity ${ }^{4} \mathrm{He}$ evaporation refrigerator. Finally, to invoke and maintain the DNP process, continuous microwave irradiation is facilitated by an EIO tube with a center frequency around $140 \mathrm{GHz}$. The geometry of the E1039 magnet homogeneity volume allowed extension of the target cell length from the typical $20 \mathrm{~mm}-40 \mathrm{~mm}$ to $80 \mathrm{~mm}$ to further increase the integrated luminosity. The target ladder was designed to accommodate up to three Kel-f target cups with elliptical cross section to match the beam profile.

The polarization measurement system is another critical element of the target infrastructure as the systematic error due to the present accuracy of the polarization measurement often becomes a limiting factor in the overall experimental results. There are several challenges specific to the SpinQuest experimental environment that particularly affect conventional nuclear magnetic resonance (NMR) based techniques. In this proceedings we would like to present a new Q-meter module, 
along with an integrated data acquisition system, which was designed by the high energy nuclear physics (HENP) team at Los Alamos National Laboratory (LANL).

\section{Liverpoool Q-meter}

The standard technique used for measuring polarization in solid polarized targets is based on the principle of a continuous wave NMR (CW-NMR). The resonance condition is normally satisfied by sweeping the RF field around the Larmor frequency of a given nucleon species, which is applied perpendicular to the axis of the static holding field. The resulting nucleon spin-flip transitions produce energy gains or losses detectable on a macroscopic level. They are usually characterized by a frequency dependent complex susceptibility instead of macroscopic magnetization,

$$
\chi(\omega)=\chi^{\prime}(\omega)-i \chi^{\prime \prime}(\omega)
$$

with an absorptive term $\chi^{\prime \prime}$ related to the polarization of the sample as

$$
P \propto \int_{\Delta \omega} \chi^{\prime \prime}(\omega) d \omega
$$

The scanning RF field is generated in the NMR coil, which is typically embedded in the target material and connected to a tuned RF-resonant circuit through a coaxial transmission cable. The circuit, a so-called Liverpool Q-meter, was developed in the late 70s and became the de facto standard for NMR-based polarization measurements in solid polarized targets [\$]]. In general, the Liverpool Q-meter is a constant current series resonance circuit which is sensitive to the changes of the NMR coil impedance induced by modifications of the material susceptibility during frequency scan. Given that the constant current condition is preserved, the actual polarization value can be extracted by integrating the real part of the Q-meter output voltage change $\Delta V(\omega)$, i.e. by extracting the area under the NMR line-shape, around center resonant frequency $\omega_{0}$. In practice, several steps are required for this procedure: (a) performing resonance and phase tuning and resonant cable length adjustment, (b) measuring and subtracting the Q-meter response in the off-resonance region of the RF sweep, (c) performing Q-meter calibration by obtaining NMR signal at conditions where the polarization is known.

While revolutionary for its time with its introduction of phase-sensitive detection and proven reliable performance over 4 decades, the Liverpool Q-meter can no longer be manufactured according to the original design [ $[$ ] , as many RF components become obsolete. Due to the high demand and reduced availability, several groups (including our team) have worked on designing a system which would replace the Liverpool Q-meter and potentially add extra functionality [ [, , $]$ ]. Some of the SpinQuest specific requirements with respect to polarization measurements include: (i) ability to resolve polarization changes in the material along the beam direction, which would require operating up to nine NMR channels not including spares; (ii) ability to resolve polarization degradation during the $\sim 5 \mathrm{~s}$ long beam spill, which requires faster data acquisition for improved NMR statistics; (iii) addressing NMR signal degradation due to the hall's radiation environment and thus increased length of the resonant cable. Therefore, we have chosen to design a modern set of NMR electronics that are less expensive and easier to manufacture in quantity, while providing new functionality beyond those of the existing Q-meters. 


\section{LANL Q-meter}

The idea behind LANL's new design was to offer a fully integrated polarization measurement system for simplified configuration and exploitation. The normal operation of the standalone Liverpool Q-meter module necessitates development of concomitant infrastructure such as water-cooled custom-made housing crate with a precision power supply, a separate low frequency (LF) amplification unit, and a choice from commercially available data acquisition (DAQ) system, which is responsible for synchronization of the RF sweep control and the corresponding triggering of the DAQ readout. The architecture of a new LANL NMR system which combines all necessary infrastructure is presented by a set of a minimum of three printed circuit boards housed in a standard $6 \mathrm{U}$ VME crate with a total capacity of up to six independent NMR channels per crate. An external frequency synthesizer is required to provide the RF power. A pair of two boards, an Analog NMR signal processor and a Digital board, forms a single NMR channel. A single 6U dual slot VME Controller board is required per VME crate for I/O operations. In general, each board design features state-of-the-art, low-cost signal components with extremely low-noise, low-drift and flat frequency response over a large frequency range.

\subsection{Analog NMR signal processor}

LANL's Analog NMR signal processor board comprises joint functionality of the Liverpool Q-meter module together with a post-stage LF amplifier typically known as a Yale-Card. This board follows the Liverpool Q-meter basic design in that it is using a phase-sensitive demodulator to measure the real part of the amplified output of the resonant part of the circuit. Similarly, an additional demodulation channel is added for magnitude detection and tuning. The board is designed to operate over a frequency range of $\sim 10 \mathrm{MHz}-250 \mathrm{MHz}$, covering the typical signals from both protons and deuterons in high magnetic fields. The printed circuit board has eight metal layers and was designed using the EAGLE CAD software. Most traces carrying RF are $50 \Omega$ striplines, providing excellent shielding. Only one inexpensive mechanical shield is required over the section containing the 1st RF amplifier. A schematic diagram of the board is shown in Fig. $\mathrm{W}$.

A number of functionally new features that were introduced in the new module include: (i) overall compact design with low power consumption ( $\sim 5 \mathrm{~W}$ per channel); (ii) resonant part of the circuit is built on a separately shielded external daughter board for easy switching to non-resonant cable mode of operation; (iii) varactor diode serves as a variable capacitor for remote digital resonance tuning which is performed by changing the tuning voltage (VTune); (iv) circuit can electronically shift the phase of an applied signal by over $180^{\circ}$ and eliminate the need for manual phase cable adjustment; (v) digital RF attenuator together with post-mixer LF amplification allows precise optimization of the overall RF gain of the system and setting the dynamic range appropriately for a given nuclear species and the filling factor of the NMR coil; (vi) diode detector is replaced with logarithmic amplifier which exhibit much better linearity and frequency response; (vii) digitally controlled DC offset subtraction. A temperature compensation circuit applies a small correction to the tuning voltage, keeping the tune stable over a large temperature range. Monolithic microwave integrated circuits (MMIC, Fig. W) and a digital attenuator amplify the signal level to that optimized for the mixer. Most of the radio frequency components were obtained from Mini-Circuits and have well specified performance. 


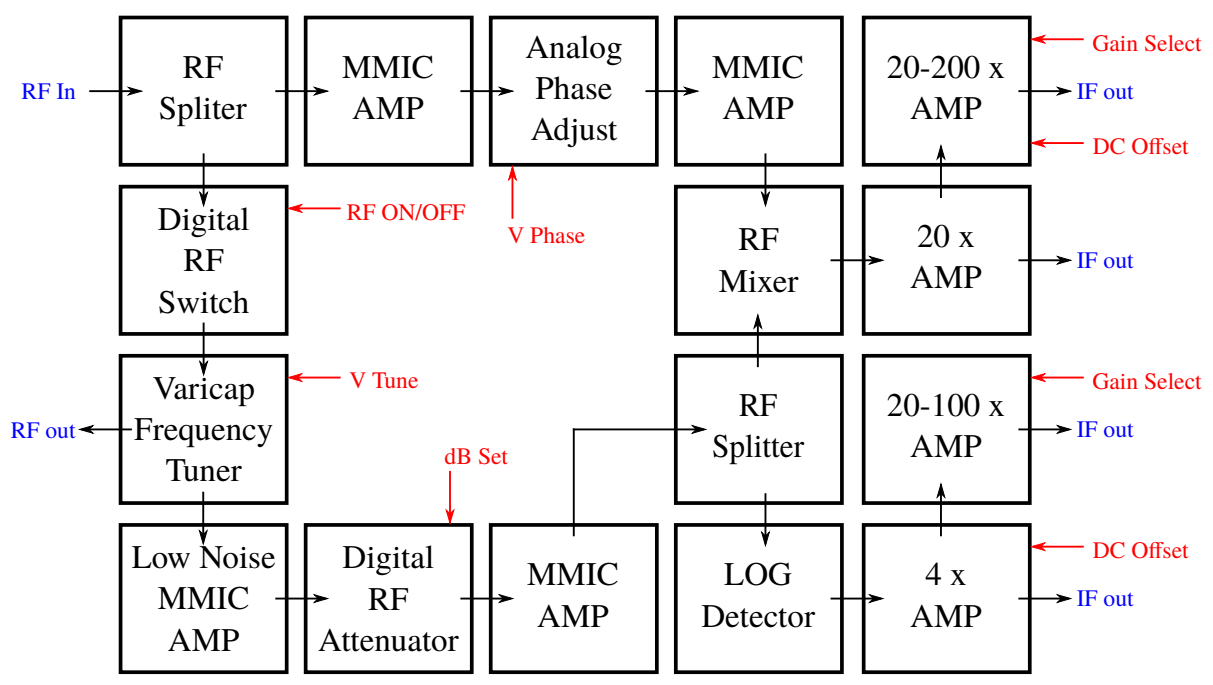

Figure 1: Block diagram of the RF section of the LANL NMR system. Inputs shown in red color indicate digitally controlled elements.

\subsection{Digital board}

The Digital Board envelops a variety of services usually implemented by relying on a mixture of a custom-made DIO controllers and industrial DAQ systems such as National Instruments products. A schematic diagram of the board is shown in Fig. $\square$. This $6 \mathrm{U}$, single slot VME card contains a 4-channel 16 bit bipolar ADC and 4-channel 16 bit bipolar DAC. The on-board 4-channel ADC unit can perform, one at a time, digitization of both demodulation channels at two different stages of amplification (see IF out locations in Fig. W) at a maximum speed of $100 \mathrm{ksps}$. One of the channels of the on-board DAC unit provides voltages for frequency modulation of the external RF synthesizer. The other DAC channels are used to set the tune, phase, and DC offset voltages.

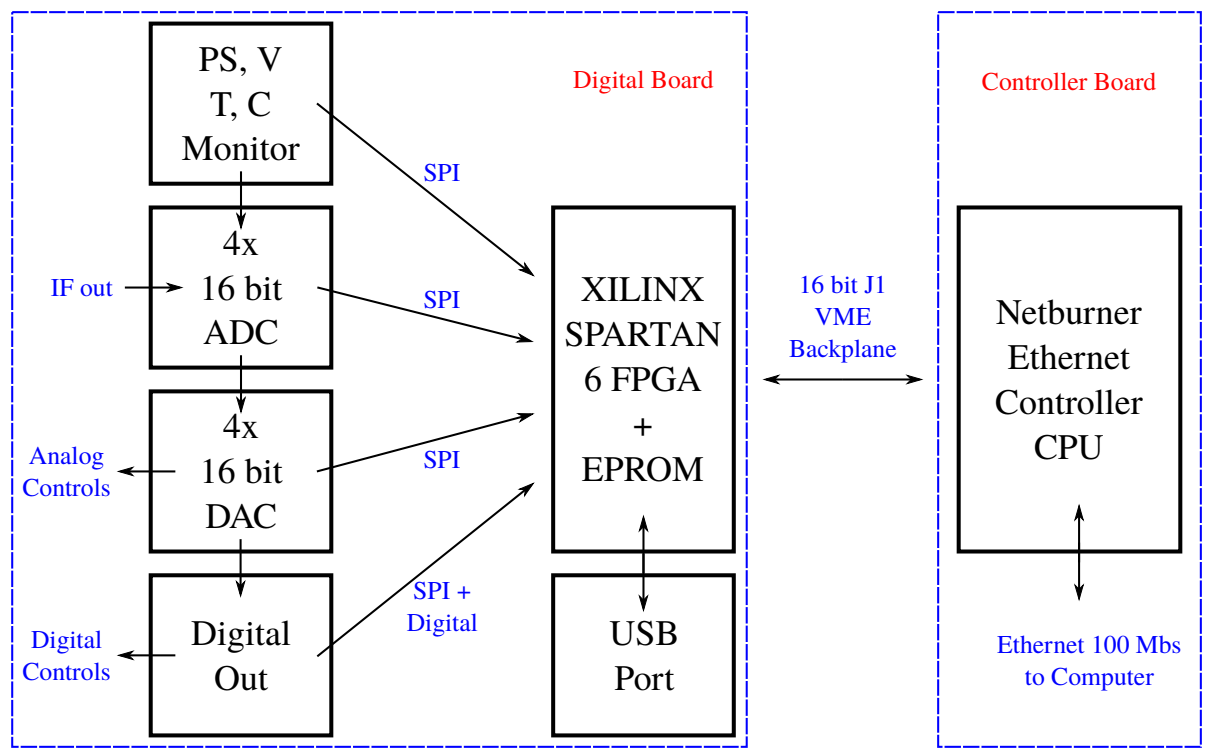

Figure 2: Block diagram of NMR digital and controller sections. 
The board also performs temperature measurements and the monitoring of the precision voltage supply (3.3V) output. A Spartan-6 field programmable gate array (FPGA) daughter-board routes the digital signals through the VME back-plane to the crate controller. The FPGA can act as either a multiplexer to switch various digital devices directly onto the VME bus or as an intelligent controller that accepts commands from the controller and then performs them independently.

\subsection{Crate controller board}

The VME controller board is based upon a Netburner system-on-a-module micro-controller contained on a daughter-card. This 32 bit processor runs a real time operating system that is stored in non-volatile program memory. Peripheral interfaces include Ethernet, serial ports, USB port and a 16 bit bi-directional data bus. The controller board buffers this data bus onto the VME backplane, creating an inexpensive yet powerful VME system. The microprocessor is programmed in $\mathrm{C}++$ with the included editor, GCC compiler and Netbutrner software libraries. Communication with a desktop computer running LabVIEW is carried over a $100 \mathrm{Mb}$ Ethernet link using telnet. The controller can be programmed over Ethernet or USB. After programming or a power cycle, the controller completes booting in just a few seconds.

\section{Comparison tests}

Besides normal performance tests, special emphasis was placed upon a precise comparison between LANL Q-meter and Liverpool Q-meter. To minimize systematic uncertainty in this comparison we developed a custom setup to reliably control and switch the signal sources between the two systems. While the comparison of the real polarization signals provides the most stringent testing framework, the cool-downs are expensive and time consuming procedures. Therefore, for a certain number of tests a surface acoustic wave resonator (a crystal box) was used to simulate a proton signal (at 5 ) on a hardware level to produce a clean narrow peak at $\sim 224.5 \mathrm{MHz}$, slightly above the proton's Larmor frequency.

\subsection{Linearity tests}

A precision tool was built for full dynamic range linearity tests. A crystal box was inductively coupled with an NMR coil to produce large initial signal. The resonant part of the circuit was common for both NMR systems, thus allowing comparison of the entirety of post-LCR electronics, eliminating tuning and $\lambda / 2$ cable effects. Two high precision 7 bit digitally controlled RF attenuators in series provide wide dynamic range coverage. A low loss electronically controlled RF switch was used for rapid switching between the LANL and Liverpool Q-Meters. An amplitude scan on the crystal signal that covered $\mathrm{a}>60 \mathrm{~dB}(\sim \times 1000)$ range was performed in $3 \mathrm{~dB}$ steps. While the systematics analysis is still underway, preliminary results (Fig. [B]) show that both systems agree at $\sim 1 \%(<2.5 \%)$ level in operational range (at the edges).

\subsection{Polarization tests}

Polarization comparison tests were carried out by observing the proton polarization signal from a $\mathrm{NH}_{3}$ sample under the normal $\left(B_{0}=5 \mathrm{~T}, T=\sim 1 \mathrm{~K}, \omega_{0}=213 \mathrm{MHz}\right)$ conditions. Both 


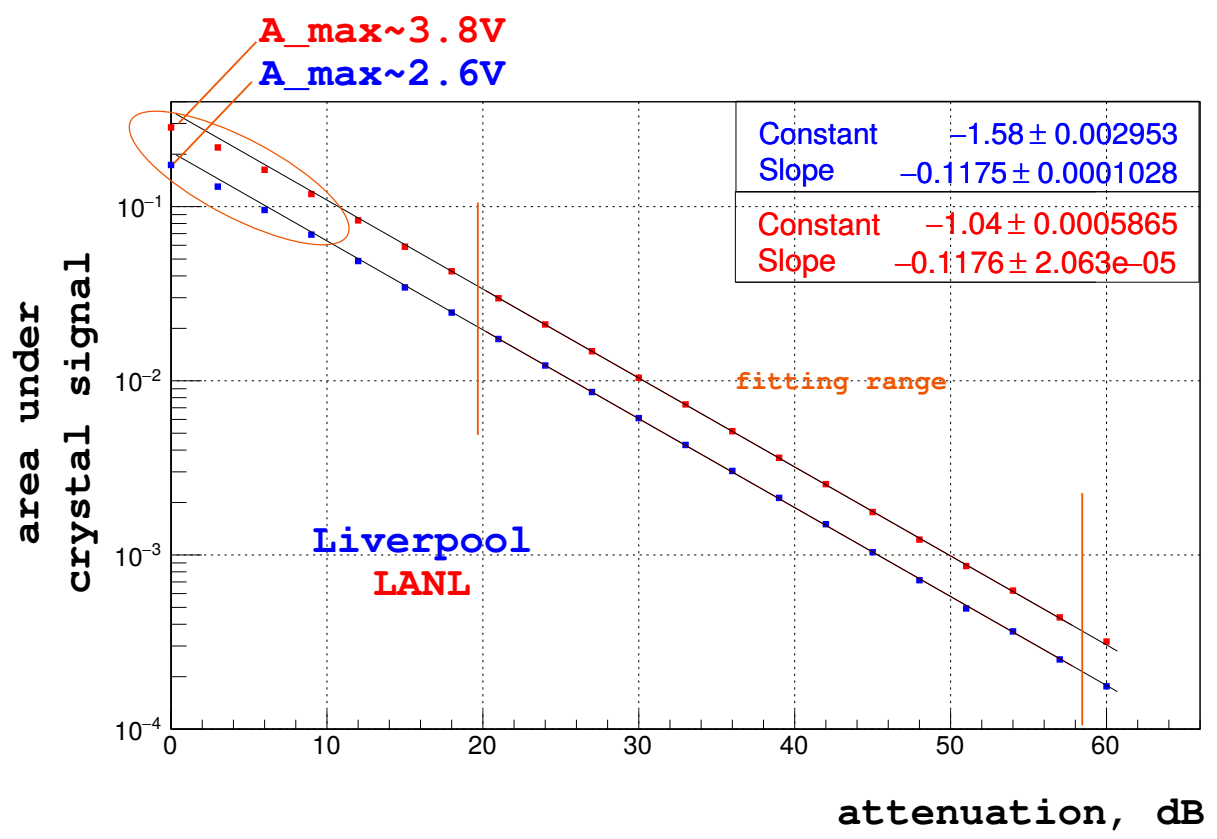

Figure 3: The area (arbitrary units) under the crystal signal extracted after polynomial Q-curve subtraction is plotted as a function of RF attenuator setting: red(blue) is LANL(Liverpool Q-meter response). The orange vertical lines indicate fitting range. The black lines indicate extrapolation into the non-linear region shown in the orange oval. Systematic uncertainties are not included.

systems shared the same NMR coil through the low loss electronically controlled RF switch. The $\lambda / 2$ cable was also common for both systems except for the small portion that was split to facilitate connection of the corresponding module.

Measurements started by performing thermal equilibrium (TE) calibration with Liverpool Qmeter. Once the online proton polarization calibration constant was obtained, the TE measurements with the LANL Q-meter were performed. The sample was then polarized positively. As polarization was ramping up, the NMR coil was rapidly switched between the two systems to acquire comparison data. Two full cycles of positive and negative polarization data were collected during the cool-down. In order to eliminate uncertainty related to ${ }^{4} \mathrm{He}$ vapor pressure measurements, preliminary analysis focused on comparing the ratio of extracted signals. Both data sets were normalized at one of the positive polarization saturation points where the signals are at the maximum and Q-curve subtraction effects are minimized. Preliminary results (Fig. 团) show that both systems agree at $\sim 1.5 \%$ level. The LANL Q-meter demonstrated similar or better $\mathrm{S} / \mathrm{N}$ performance. The actual signal shapes are in the excellent agreement.

The option of selecting resonant or non-resonant cable (i.e. cold NMR [Q]) modes of operation was a prerequisite at the development stage of the LANL Q-Meter. Since the cold NMR operation is typically required for small deuteron signals, a special variant of the Analog Board can be produced with a minor component modifications. These modifications are mostly required to accommodate electronic phase adjustment for lower frequency operation. The functionality of the 32.7 MHz adjusted cold NMR setup was tested in a parasitic run during the cool-down conducted at University of Virginia. An enhanced polarization signal from a deuterated butanol sample was 


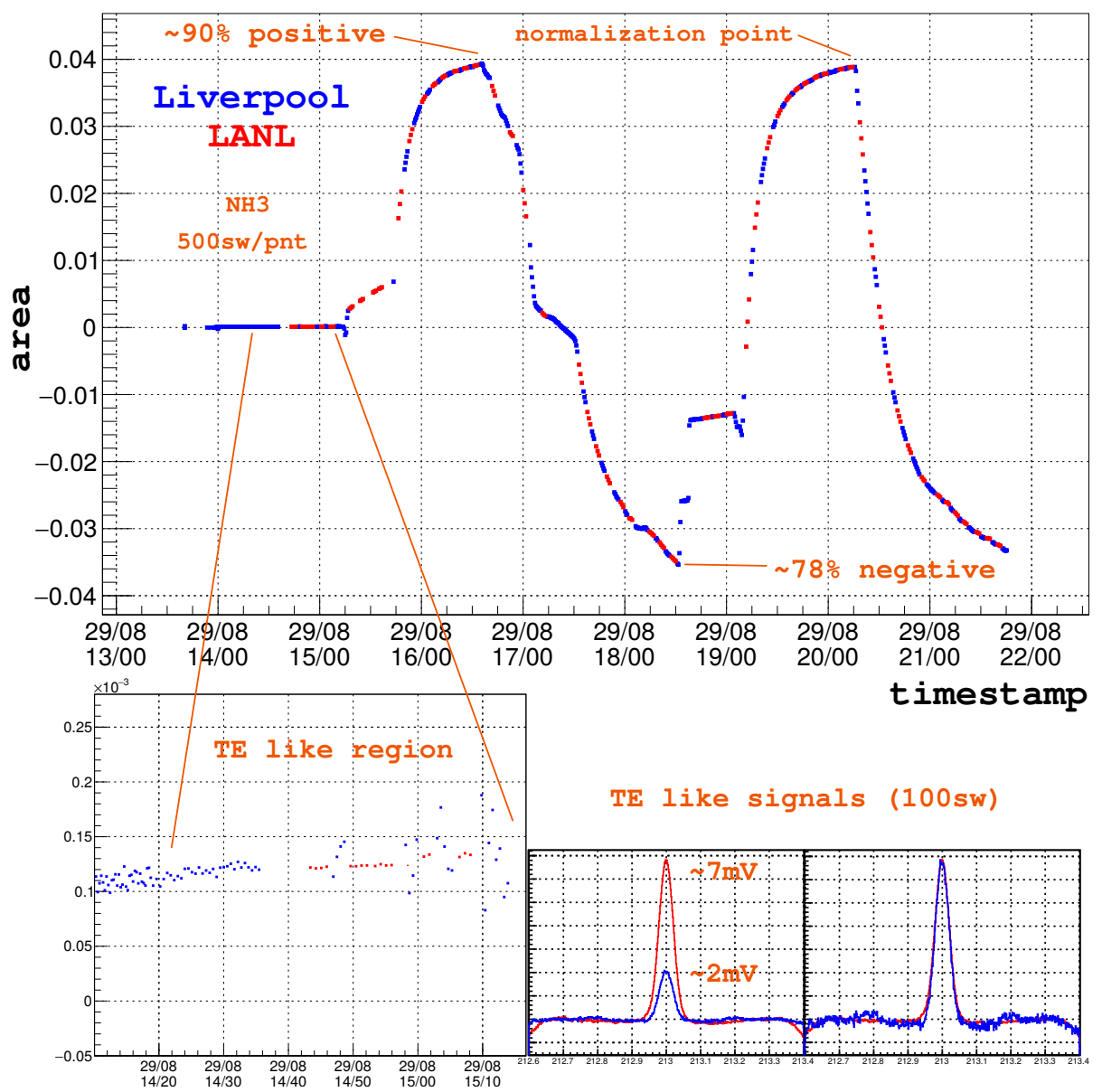

Figure 4: The area (arbitrary units) under the proton polarization signal extracted after standard Q-curve subtraction is plotted as a function of time: red(blue) is LANL(Liverpool Q-meter response). The calibration constant is from the preliminary online online analysis of the TE signal. Top: two cycles of polarization change; Bottom left: zoomed in TE region; Bottom right: comparison of the raw (left) and normalized (right) signals from TE region. Systematic uncertainties are not included.

observed (Fig. (5) at 5T. Due to the limited cool-down time, RF gain optimization has not been performed for the deuteron TE signal detection.
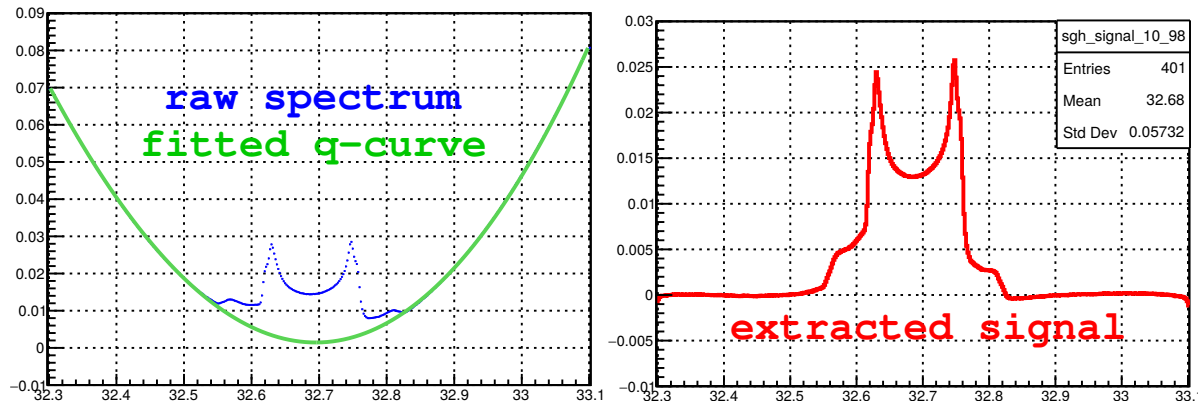

Figure 5: The enhanced polarization signal from a deuterated butanol sample during cold NMR mode of operation of the LANL Q-Meter. 


\section{New capabilities}

A rigorous theoretical treatment of the series resonant type Q-Meter, as well as a thorough discussion of the well-known limitations inherent to the Liverpool Q-meter design, were made in a number of papers [10, $\square]$ and during a dedicated workshop on NMR techniques in polarized targets organized at UVA in 1998. While the underlying effects are complex and often intertwined, the outlined difficulties can be loosely categorized as:

(a) detection of the small (quadruple broadened deuteron) signals residing on the large background;

(b) nonlinearities arising from detection of very large (high field and coil filling factor) signals;

(c) resonant cable effects that may cause (in general both proton and deuteron) signal instabilities.

The measurements of the deuteron TE signal correspond to conditions of item (a), where the ratio of the Q-curve depth to the size of the signal can be as large as 800 []. The plethora of related issues, external noise, DC level change, tune drifts, etc was partially resolved by increasing the statistics of the measurements and raising the overall Q-meter amplification to improve the $\mathrm{S} / \mathrm{N}$ ratio. Two methods for background reduction was offered by [[12, [13]: synchronous resonance tuning (SRT) and synchronous phase tuning (SPT). In both cases, dynamically controlled hardware is required in order to track the capacitance and phase at each frequency step of the RF sweep, which would flatten the background in the absence of the $\lambda / 2$ cable. The SRT was initially realized in Dubna and later at PSI [[]] while the usage of SPT has not been tried yet to our knowledge. The new LANL Q-Meter comes with a built-in hardware, i.e. varactor (SRT) and phase shifter (SPT), with the potential to realize both of these functionalities.

To demonstrate the nonlinear effects which are characteristic to conditions of item (b), authors in [U] perform series expansion of the real part of the output RF voltage and show that the nonlinear terms may, under certain conditions, lead to asymmetric polarization values. This issue was normally addressed by applying appropriate numerical corrections. Both works [एँ, प]] conclude that the in-phase detection of the Liverpool Q-meter is insufficient for reliable determination of nuclear polarization and that the measurements of the imaginary part of the signal would provide unambiguous extraction. The ultimate response to this problem was suggested by G. Court, with the idea of a fully digital fast-ADC based Q-meter for RF waveform digitization and offline extraction of both the absorptive and dispersive parts of the nuclear susceptibility. The alternative suggestion [ए]] was to design a Q-Meter with two phase sensitive demodulators (PSD), performing the equivalent function. Since the LANL Q-Meter comes with a digitally controlled phase shifter, it offers yet another possibility for measuring the imaginary part of the signal. It is possible to use the single PSD on the LANL Analog Board and sequentially switch the predetermined phase tune from real to imaginary detection on every RF sweep.

Cable effects listed in item (c) are one of the biggest contributors to polarization uncertainties. Particularly in the circumstances where the $\lambda / 2$ cable length cannot be minimized, cable attenuation, dielectric properties and temperature dependence, large asymmetric reactive impedance in 
the off-resonance region of the RF sweep, and induced noise all become defining factors of NMR signal quality. Another important, and often overlooked, factor in proper usage of series Q-meter, particularly at high RF frequencies, is operator errors in $\lambda / 2$ length adjustments which may lead to a false LCR tune. While comparing the Liverpool and LANL Q-meters, it was noticed that both systems are extremely sensitive to even small variations of the $\lambda / 2$ length. As it was noted in [[4]], extra cable length is equivalent to adding a parallel capacitance across the coil. Such issues are completely obviated by placing the entire resonant circuit in the proximity of the target material (in the cryostat) and thus completely eliminating the need for a resonant $\lambda / 2$ cable. This configuration, known as Cold NMR, has become increasingly popular in the recent experiments. Another benefit of the Cold NMR is the reduction of the Johnson noise in the cooled LCR part of the Q-meter. As was mentioned earlier, the LANL Q-Meter is proven to operate in both configurations.

\section{Conclusion}

The high energy nuclear physics team at LANL has developed a new Q-meter for the measurements of proton and deuteron polarization in solid polarized targets. This new cost efficient fully integrated compact system includes NMR analog electronics, a fast digitizer and single board microprocessor with Ethernet interface. The system provides a variety of built-in hardware solutions previously inaccessible in a single setup. This new functionality opens an opportunity to increase the precision and accuracy of NMR based polarization measurements. Practical implementation of these newly developed capabilities is the next important step of this program.

\section{Acknowledgments}

We would like to thank the solid polarized target group at the University of Virginia for continuous support, useful discussions and for providing required infrastructure for tests and measurements. We also would like to thank the solid polarized target group at the University of New Hampshire for testing out our system.

\section{References}

[1] D. Geesaman, P. Reimer, C. Brown, D. Christian, M. Diefenthaler, J. C. Peng et al., Letter of Intent for a Drell-Yan Experiment with a Polarized Proton Target, .

[2] D. G. Crabb, C. B. Higley, A. D. Krisch, R. S. Raymond, T. Roser, J. A. Stewart et al., Observation of a 96\% proton polarization in irradiated ammonia, Phys. Rev. Lett. 64 (1990) 2627.

[3] D. Crabb and D. Day, The Virginia/Basel/SLAC polarized target: operation and performance during experiment E143 at SLAC, Nuclear Instruments and Methods in Physics Research Section A: Accelerators, Spectrometers, Detectors and Associated Equipment 356 (1995) 9.

[4] D. G. Crabb and W. Meyer, SOLID POLARIZED TARGETS FOR NUCLEAR AND PARTICLE PHYSICS EXPERIMENTS, Annual Review of Nuclear and Particle Science 47 (1997) 67 [https://doi.org/10.1146/annurev.nuc1.47.1.67].

[5] G. Court, D. Gifford, P. Harrison, W. Heyes and M. Houlden, A high precision Q-meter for the measurement of proton polarization in polarised targets, Nuclear Instruments and Methods in Physics 
Research Section A: Accelerators, Spectrometers, Detectors and Associated Equipment 324 (1993) 433 .

[6] G. Court, The development of NMR techniques for the high precision measurement of target polarisation, Nuclear Instruments and Methods in Physics Research Section A: Accelerators, Spectrometers, Detectors and Associated Equipment $\mathbf{5 2 6}(2004) 65$.

[7] J. Herick, Development of a new Q-meter module, PoS PSTP2015 (2016)011.

[8] J. D. Maxwell, NMR Measurements for Solid Polarized Targets at Jefferson Lab, EoS SPIN2018 (2019) 102

[9] G. Court, M. Houlden, S. Bültmann, D. Crabb, D. Day, Y. Prok et al., High precision measurement of the polarization in solid state polarized targets using NMR, Duclear Instruments and Methods in Physics Research Section A: Accelerators, Spectrometers, Detectors and Associated Equipment $\mathbf{5 2 7}$ (2004)253.

[10] Y. Kisselev, C. Dulya and T. Niinikoski, Measurement of complex RF susceptibility using a series Q-meter, Nuclear Instruments and Methods in Physics Research Section A: Accelerators, Spectrometers, Detectors and Associated Equipment 354 (1995) 249.

[11] T. Niinikoski, Topics in NMR polarization measurement, Nuclear Instruments and Methods in Physics Research Section A: Accelerators, Spectrometers, Detectors and Associated Equipment 356 (1995) 62

[12] G. R. Court and M. A. Houlden, Possible Methods to Reduce the size of the background signal in continuous wave Q-meters, Proceedings of the Workshop on NMR in Polarized Targets, UVA Charlottesville, VA (1998) 7.

[13] P. Hautle, Detection of small NMR signals, Proceedings of the Workshop on NMR in Polarized Targets, UVA Charlottesville, VA (1998) 54.

[14] G. R. Court and M. A. Houlden, The effect of Coil Stray Capacity on the Performance of a Series Tune NMR Q-meter, Proceedings of the Workshop on NMR in Polarized Targets, UVA Charlottesville, $V A$ (1998) 1. 